\title{
Prevalência de doenças sistêmicas entre os pacientes atendidos na Clínica Odontológica da Faculdade Católica Rainha do Sertão no município de Quixadá-CE
}

Prevalence of systemic diseases among patients attended at the Dental Clinic of Catholic College of the Rainha do Sertão in Quixadá-CE Prevalencia de enfermedades sistémicas entre los pacientes atendidos en la
Clínica Odontológicaen Escuela Católica de Odontología en Quixadá-CE

\author{
Lizianea de Aragão GADELHA ${ }^{1}$ \\ Rodrigo CAPALBO-da-SILVA ${ }^{2}$ \\ Valthierre Nunes de LIMA ${ }^{2}$ \\ Gustavo Antonio Correa MOMESSO ${ }^{2}$ \\ Sormani Bento Fernandes de QUEIROZ ${ }^{2}$ \\ Francisley Ávila SOUZA ${ }^{2}$
}

${ }^{I}$ Dentist by Catholic University Center of Quixadá - UNICATÓLICA 63900-257 Quixadá, Ceará, Brazil

${ }^{2}$ Department of Surgery and Integrated Clinic, Aracatuba Dental School- UNESP, 16015-490 Aracatuba, São Paulo, Brazil.

\begin{abstract}
Resumo
As emergências médicas no consultório odontológico são raras, todavia, pacientes com alterações sistêmicas são mais propensos a estas requerendo de atendimento especial do cirurgião-dentista, especialmente os portadores de alterações cardiovasculares e endócrinas, as quais têm forte impacto na saúde oral e representam uma das principais causas de óbito na população adulta brasileira. Diante da importância da atuação do odontólogo em pacientes com comprometimento sistêmico, o estudo denota como objetivo avaliar a prevalência de enfermidades ou condições sistêmicas encontradas no atendimento odontológico. Para o desenvolvimento desse trabalho foi realizado um levantamento nos prontuários de 1600 pacientes atendidos na clínica de odontologia da Faculdade Católica Rainha do Sertão no Munícipio de QuixadáCe. Do total de prontuários analisados foi encontrado um percentual de 6,6\% de alterações sistêmicas, sendo $2,8 \%$ alterações cardiovasculares, $0,7 \%$ diabetes e 3,1\% outras alterações sistêmicas. Concluiu-se que, das alterações cardiovasculares, a hipertensão foi à patologia de maior prevalência. Foram encontrados $48,1 \%$ de pacientes em acompanhamento médico sendo o gênero mais acometido o feminino com $63 \%$ e uma faixa etária de 30 a 39 anos de idade.

Descritores: Doença; Comorbidade; Diabetes Mellitus; Hipertensão.
\end{abstract}

\begin{abstract}
The medical emergency in the dental office are rare, however, patients with systemic changes are more likely to this requiring are special treatment of dental surgeon, especially the disease carrier of cardiovascular and endocrine, which have a very strong impact on oral health and represent one of major cause of obit in the adult Brazilian population. The importance of the current conditions from the dentist`s evaluation in patients with systematic disease the study indicate like objective evaluate the disease prevalence or systematic conditions found in dental treatment. To the development of this work was made a cadastral survey from 1600 medical records in dental clinic of Catholic College of the Rainha do Sertão in Quixadá County, State of CE. From all the analyzed records was found 6,6\% percent of systemic diseases, with $2,8 \%$ cardiovascular diseases, $0,7 \%$ diabetes and $3,1 \%$ of others systemic diseases. It was concluded that of $48,1 \%$ of the patients in medical treatment, $63 \%$ was the female gender with and age group from 30 to 39 years old.

Descriptors: Disease; Comorbidity; Diabetes Mellitus; Hypertension.
\end{abstract}

\section{Resumen}

Las emergencias médicas en el consultorio dental son raros, sin embargo, los pacientes con alteraciones sistémicas son más propensos a éstos requieren cuidados cirujano dental especial, especialmente aquellos con trastornos cardiovasculares y endocrinos, que tienen un fuerte impacto en la salud oral y representan una de las principales causas de muerte en la población adulta brasileña. Dada la importancia del trabajo del dentista en pacientes con afectación sistémica, el estudio indica para evaluar la prevalencia de enfermedades o afecciones sistémicas que se encuentran en el cuidado dental. Para el desarrollo de este trabajo se llevó a cabo una encuesta sobre las historias clínicas de 1.600 pacientes tratados en la clínica dental de la zona de influencia de la reina de la escuela católica en municipio de QuixadáCE. De las historias clínicas analizadas se encontró un porcentaje del 6,6\% de los cambios sistémicos, con 2,8\% para alteraciones cardiovasculares, $0,7 \%$ para la diabetes y 3,1\% para otros cambios sistémicos. Se concluyó que dentro de las alteraciones sistémicas, la hipertensión fue la patología más prevalente. $48,1 \%$ de los pacientes se encontraron en la atención médica y las mujeres fueran más afectadas (63\%) y con edad de 30 a 39 años.

Descriptores: Enfermedad; Comorbidad; Diabetes Mellitus; Hypertensión.

\section{INTRODUÇÃO}

Emergências médicas podem ocorrer durante o atendimento odontológico, apesar de raras, e sendo os pacientes portadores de alterações sistêmicas mais propensos a essa ocorrência, estes requerem de atendimento especial do cirurgião-dentista ${ }^{1}$. É essencial que o profissional esteja ciente da conduta a ser tomada diante de um eventual quadro de emergência e tenha conhecimentos necessários para conduzir adequadamente o tratamento sem causar danos ao paciente ou comprometer o desenvolvimento do caso clínico $^{2}$, sendo imperativo que o odontólogo esteja apto a avaliar o estado geral do paciente a fim de evitar ou contornar complicações indesejáveis ${ }^{3,4}$. 
Dentre as enfermidades sistêmicas que requer atenção especial do cirurgião-dentista, destacam-se as doenças endócrinas, como a Diabetes Mellitus ${ }^{5}$. A diabetes afeta cerca de 17 em cada mil pessoas em uma faixa etária de 25 a 44 anos, e 79 indivíduos em cada mil, com idade acima de $65 \operatorname{anos}^{6}$. Nesse contexto, aproximadamente $3 \%$ a $4 \%$ dos pacientes adultos que se submetem ao tratamento odontológico possuem Diabetes, sendo imperativo um completo planejamento para o correto manejo clínico desses pacientes com o intuito de reduzir e/ou evitar possíveis complicações de tal doença ${ }^{7,8}$.

As doenças cardiovasculares permanecem sendo as principais causas de morte na população e, corroborado por diversos autores, a doença coronária é entre, as doenças cardiovasculares, a de maior expressão tanto no nível de prevalência quanto no de morbimortalidade ${ }^{8,9}$. É representada por um amplo espectro clínico que varia desde a doença arterosclerótica assintomática, passando por quadros de angina estável até quadros mais graves de infarto agudo do miocárdio, sendo imprescindível a intervenção cautelosa do cirurgião-dentista ${ }^{9,10}$.

Tendo em vista que os pacientes com comprometimento sistêmico são pacientes diferenciados que necessitam de um manejo clínico compatível com suas necessidades, é objetivo desse trabalho elucidar a prevalência das principais doenças sistêmicas avaliadas em pacientes atendidos no Complexo Odontológico da Faculdade Católica Rainha do Sertão localizada no município de Quixadá-CE, com ênfase nas doenças cardiovasculares e endócrinas.

\section{MATERIAL E MÉTODO}

Para o desenvolvimento deste trabalho, foi realizado um levantamento em 1600 prontuários dos pacientes atendidos, no período de 2007 a 2011, no Complexo Odontológico São João Calábria da Faculdade Católica Rainha do Sertão. Os prontuários foram selecionados aleatoriamente e identificados os pacientes portadores de patologias, com ênfase, nas doenças cardiovasculares e endócrinas.

O projeto de pesquisa em questão foi submetido ao Comitê de Ética em Pesquisa com Seres Humanos da Faculdade Católica Rainha do Sertão - FCRS e recebeu parecer favorável ao seu desenvolvimento.

Os dados obtidos foram compilados e apresentados na forma de tabelas e gráficos, avaliando a prevalência de doenças de base, entre os pacientes atendidos, sendo os mesmos dados analisados quantitativamente e transformados em porcentagem para facilitar a discussão e a comparação dos resultados com os relatos da literatura, o que corrobora com Carvalho, Mosele ${ }^{11}$.

Os fatores considerados para a avaliação incluíram idade, gênero, manifestações sistêmicas em antecedentes familiares, principais enfermidades e condições sistêmicas que afetam os pacientes, tais como: hipertensão, doenças cardíacas, doenças endócrinas, as quais foram correlacionadas com a faixa etária e o gênero dos pacientes.

\section{RESULTADOS}

Foram analisados 1600 prontuários clínicos e, destes, 106 apresentaram respostas positivas a enfermidades ou condições sistêmicas $(6,62 \%)$. Dentre os prontuários avaliados, em alguns foi observada mais de uma resposta positiva referente às enfermidades em questão. As enfermidades ou condições sistêmicas de maior prevalência considerando a amostragem de 106 casos positivos estão demonstradas na Figura 1. Destes, 55 (3,5\%) apresentaram alterações cardiovasculares em associação com a diabetes e $51(3,1 \%)$ outras alterações sistêmicas.

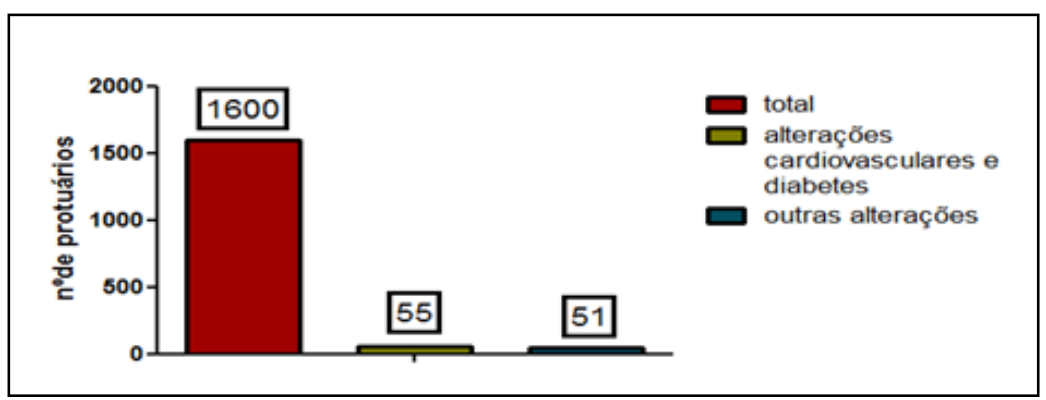

Figura 1: Enfermidades ou condições sistêmicas mais prevalentes n população avaliada. (Resultado absoluto encontrado a partir da amostra positiva).

$\mathrm{Na}$ Figura 2, verificam-se as enfermidades encontradas no presente estudo, relacionando-as com o total geral de prontuários clínicos.

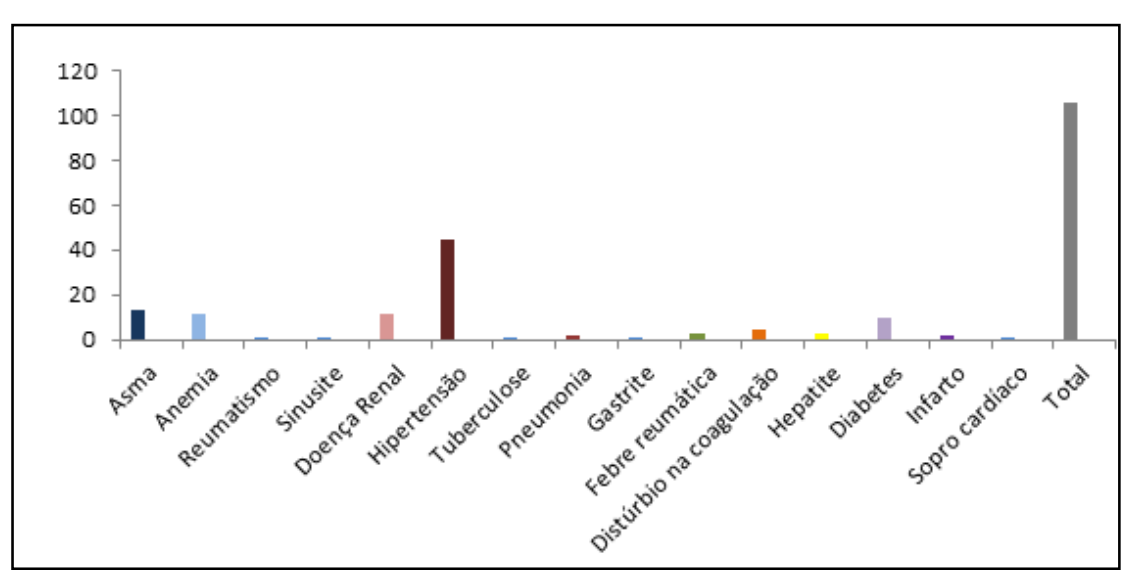

Figura 2: Total de enfermidades ou condição sistêmica encontradas, correlacionando-as, com o total geral de prontuários avaliados.

As enfermidades de maior prevalência podem ser analisadas na Figura 3.

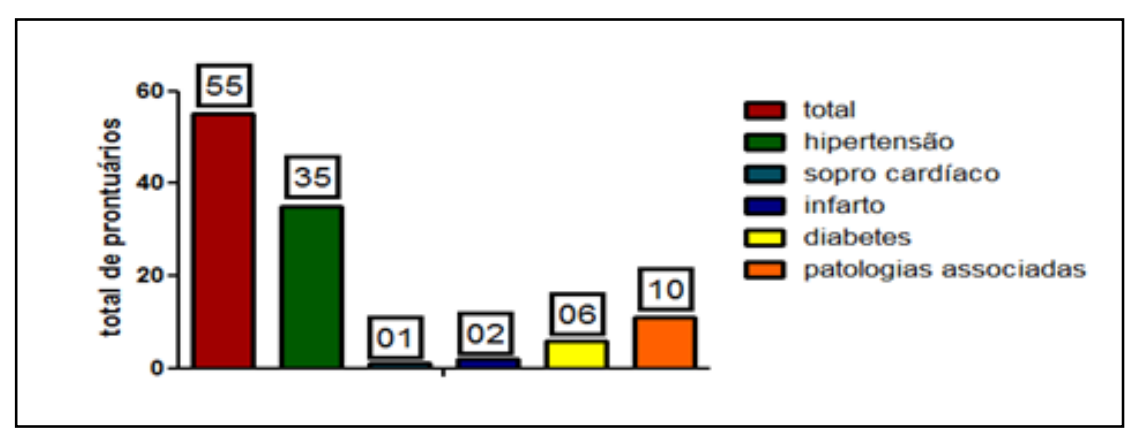

Figura 3: Enfermidades de maior prevalência expressa em número total de prontuários clínicos analisados no estudo.

\section{- Faixa etária}

Os pacientes foram divididos em 8 faixas etárias, desde os 10 anos a 89 anos de idade. A faixa etária que apresentou maior número de prontuários com respostas positivas às enfermidades sistêmicas foi a de 30-39 anos e a de menor prevalência foi a faixa etária de 10 a 19 anos de idade. Alguns prontuários apresentaram mais de uma alteração sistêmica.

Levando-se em consideração a faixa etária na prevalência das enfermidades, foi encontrado 9 pacientes com enfermidades de 30 a 39 anos de idade o que representou a maior prevalência da população estudada. A faixa de 10-19 anos correspondeu a 1 paciente; 6 pacientes de 20-29; 8 pacientes de $40-49$ e de $50-59$ anos; 5 pacientes 60-69; 6 pacientes de $70-79$ e 3 pacientes de $80-89$. É 
importante ressaltar que em alguns prontuários não foi constatado a idade dos pacientes.

As respostas afirmativas a enfermidades sistêmicas correlacionadas com a idade estão apresentadas na Figura 4.

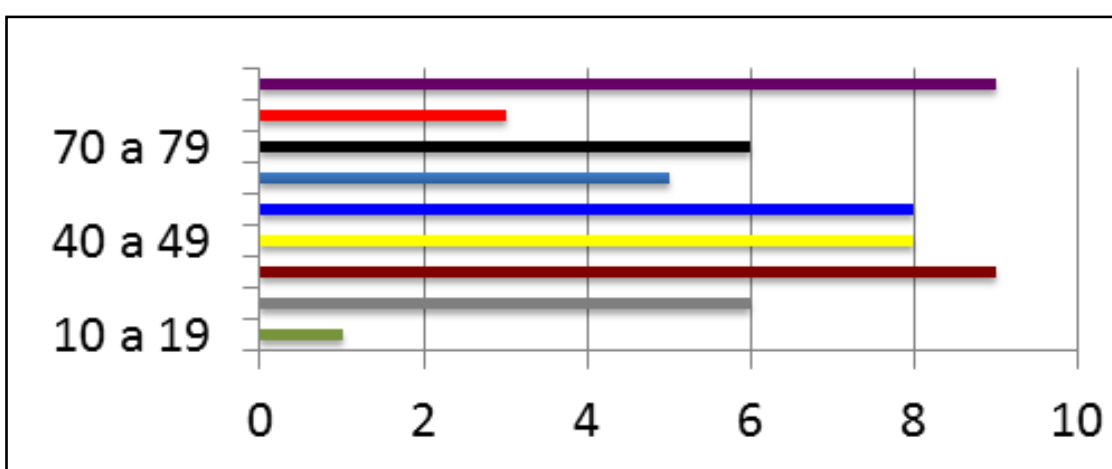

Figura 4: Prevalência de respostas positivas em relação ao total de prontuários analisados em cada faixa etária.

\section{- Relação do gênero com as condições encontradas}

Levando-se em consideração que as enfermidades de maior prevalência foram as doenças cardiovasculares e diabetes, correlacionando-as com o gênero, foi encontrado 20 prontuários com resposta positiva do gênero masculino, correspondendo a $37 \%$, enquanto 35 foi do sexo feminino, o que representa $63 \%$ dos prontuários analisados (Figura 5).

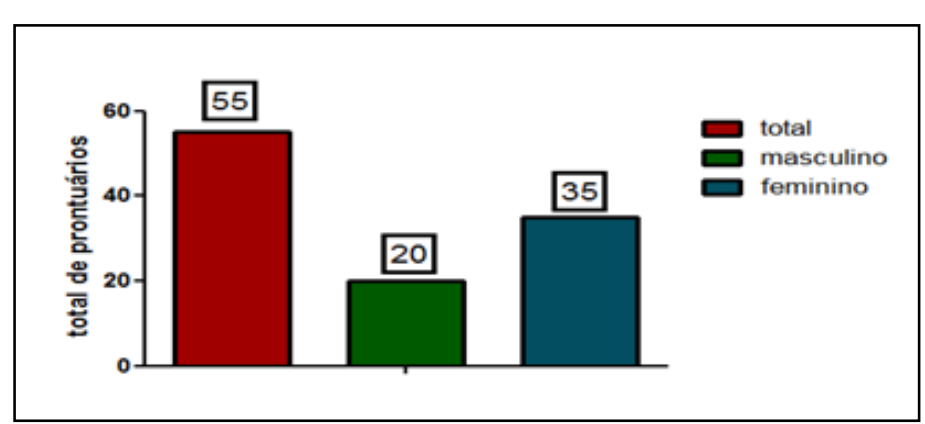

Figura 5: Distribuição das enfermidades cardiovasculares e diabetes correlativo com o gênero.

Este estudo também observou nos prontuários, se os pacientes estavam sob uso de medicação, acompanhamento médico e a correlação com antecedentes familiares (Tabela 1).

Tabela 1. Correlação das respostas positivas das condições sistêmicas quanto ao uso de medicação, acompanhamento médico e antecedentes familiares

\begin{tabular}{|c|c|c|c|}
\hline \multicolumn{4}{|c|}{ Total de pacientes } \\
\hline \multirow[b]{2}{*}{ Uso de medicamentos } & Sim & \multicolumn{2}{|c|}{ Não informou } \\
\hline & $39(70,9 \%)$ & \multicolumn{2}{|c|}{$16(29,1 \%)$} \\
\hline \multirow{2}{*}{$\begin{array}{l}\text { Acompanhamento } \\
\text { médico }\end{array}$} & Sim & Não & Não informou \\
\hline & $23(41,8 \%)$ & $16(20,1 \%)$ & $16(20,1 \%)$ \\
\hline \multirow{2}{*}{ Antecedentes familiares } & Sim & Não & Não informou \\
\hline & $34(61,8 \%)$ & $05(9,1 \%)$ & $16(20,1 \%)$ \\
\hline
\end{tabular}

\section{DISCUSSÃO}

O tempo médio de vida da população brasileira teve um aumento substancial nos últimos anos e, como consequência, a demanda de pacientes nos consultórios odontológicos tornou-se significativa, principalmente, por pessoas de mais idade. Este aumento requer do cirurgiãodentista, coerência para uma abordagem transdisciplinar, principalmente, em pacientes com enfermidades ou condições sistêmicas ${ }^{12}$.

Sonis et al. ${ }^{13}$ relataram que há negligência por parte dos profissionais, quanto ao preenchimento adequado de prontuários, dado denotado em alguns trabalhos, o que corrobora com o presente estudo, uma vez que observou-se que determinadas informações, acerca das enfermidades, foram pobremente detalhadas, bem como a omissão de informações por parte dos pacientes, como coloca Santos e colaboradores em $2009^{14}$

O presente estudo analisou 1600 prontuários e, destes, foram encontrados $106(6,6 \%)$ com resposta positiva às enfermidades sistêmicas. Esse resultado vai a confronto com a prevalência encontrada por Carvalho e Mosele em $2012^{11}$, o que pode ser explicado e corroborado, principalmente, por omissão da condição sistêmica, por parte dos pacientes, seja por ausência do conhecimento da condição clínica ou em virtude de medo de futuras intervenções médicas ${ }^{12,13}$.

Dentre as enfermidades de maior prevalência, destacaram-se as condições cardiovasculares, principalmente a hipertensão e as alterações endócrinas, a diabetes, as quais foram encontradas em um percentual de 3,5\% das respostas positivas, com um total de 35 prontuários de pacientes hipertensos e 6 de pacientes com diabetes, dado corroborado por Gaetti-Jardim et al. ${ }^{10} \mathrm{em}$ seu estudo. Carvalho e Mosele ${ }^{11}$ observaram que as doenças cardiovasculares denotaram uma maior prevalência na faixa etária de 50-59 anos o que confronta com o presente estudo, na qual a faixa etária de 30-39 anos e o gênero feminino (63\%) predominou.

As doenças do aparelho circulatório constituem as principais causas de morte na população adulta brasileira e de internações hospitalares no âmbito do Sistema único de Saúde (SUS), sendo a hipertensão arterial o principal fator de risco modificável para doenças coronarianas, doenças cerebrovasculares, insuficiência cardíaca, dentre outras ${ }^{16}$ Esse dado é corroborado com o presente estudo, visto ter sido a hipertensão a patologia de maior prevalência, dado que evidencia a importância da atuação do cirurgião-dentista em pacientes com determinada condição sistêmica a fim de minimizar e, principalmente, evitar complicações clínicas.

Quanto a outras alterações cardiovasculares, apenas infarto e sopro cardíaco foram encontrados nos prontuários avaliados. Não houve nenhum dado em relação a angina, bradicardia e arritmias. Sonis et al. ${ }^{13}$ não relatam a prevalência de tais enfermidades, mas enfatizam os cuidados que o odontólogo deve ter ao receber pacientes com tais patologias. O atendimento de um indivíduo portador de comprometimentos cardiovasculares requer um atendimento diferenciado, constituindo um fator de suma importância, a duração da consulta, que deve ser curta, além, da posição supina em que dever ficar estes pacientes, em virtude do comprometimento respiratório, que estes podem apresentar ${ }^{5}$.

$\mathrm{O}$ diabetes representou $0,7 \%$ do total de prontuários avaliados, no total de 10 pacientes, apresentando predominância no gênero feminino e com prevalência na faixa etária de 30-39 anos. De acordo com Carvalho e Mosele $^{11}$, nos casos de pacientes diabéticos controlados, estes são considerados como pacientes normais, devendo-se intervir em horários próximos ao desjejum e à ingestão de medicamento, quando fizer o uso deste, de preferência no primeiro horário do dia, exaltando a importância da antibioticoterapia nas intervenções clínicas, principalmente cirúrgicas, preventivamente.

A associação de enfermidades sistêmicas requer do profissional odontólogo uma completa anamnese e história pregressa do paciente. A elevada prevalência de patologias associadas $(20 \%)$ encontradas no presente estudo corrobora com diversos autores ${ }^{13,16}$ os quais também encontraram elevados percentuais de enfermidades sistêmicas associadas, principalmente com a hipertensão arterial, destacando a 
importância da atuação transdisciplinar do cirurgião-dentista com completa anamnese e planejamento do caso clinico ${ }^{16}$.

Ao se prescrever determinados medicamentos, devem-se considerar a metabolização e excreção da droga, que podem ser comprometidas em pacientes com alterações hepáticas e renais ${ }^{12}$. Foi encontrado no presente estudo prontuários com resposta positiva à doença renal e a associação desta com a diabetes mellitus, no entanto, esse resultado não configurou elevadas taxas de prevalência, todavia, a intervenção odontológica nesse grupo de pacientes requer uma abordagem complexa que vai desde a anamnese ao pré, trans e pós-cirúrgico, com o intuito de evitar e/ou minimizar complicações sistêmicas ${ }^{16}$.

Outra enfermidade encontrada no presente estudo foi a febre reumática, com maior incidência no gênero feminino. A intervenção de procedimentos cirúrgicos em pacientes com história de febre reumática requer atenção especial do cirurgião-dentista, quanto à antibioticoterapia prévia com o intuito de evitar complicações infecciosas ${ }^{17}$.

Neste estudo, o gênero predominante para a presença de enfermidades sistêmicas foi o gênero feminino com um percentual de $63 \%$ de respostas positivas do total de 106 prontuários com resposta positiva para patologias, dado este, corroborado por Carvalho e Mosele ${ }^{11}$ e Queiroz et al ${ }^{12}$. Todavia, Gaetti-Jardim et $\mathrm{al}^{10}$, encontrou em seu estudo uma maior prevalência de condições patológicas no sexo masculino $(64,57 \%$ dos pacientes). Alguns autores corroboram esse achado em virtude da pesquisa ter sido realizada em âmbito hospitalar, o que é sabido, que o gênero masculino denota maior prevalência nos politraumas ${ }^{10,11,13}$.

A utilização de medicamentos para o controle de manifestações sistêmicas, antecedentes familiares e o acompanhamento médico desses pacientes são fatores de suma importância para intervir clinicamente de forma segura. Na literatura revisada, não foi encontrada nenhuma menção a esses aspectos, porém, em nossa casuística observamos que $70,9 \%$ dos pacientes relataram usar medicamentos para o controle de suas doenças sistêmicas, enquanto que apenas $41,8 \%$ têm acompanhamento médico regular e 61,8\% apresentaram antecedentes familiares. Esses dados referem à importância de haver uma interação entre médico e cirurgião-dentista, para assegurar a saúde do paciente e evitar interações medicamentosas indesejáveis, bem como anamnese precisa e completa ${ }^{18}$.

\section{CONCLUSÃO}

Pacientes com enfermidades sistêmicas são mais propensos a complicações que possam vir a ocorrer durante o atendimento odontológico, principalmente, em intervenções cirúrgicas. Diante dos dados compilados no presente trabalho, a hipertensão e diabetes configurou uma grande prevalência na população de estudo, e visto que estas podem interferir de maneira desfavorável ao manejo clínico do paciente, conclui-se, que intervenções odontológicas e/ou cirúrgicas, requerem do cirurgião-dentista completa anamnese e conhecimento científico para intervir de forma segura e eficaz no tratamento clínico.

\section{REFERÊNCIAS}

1. Freitas R. Tratado de cirurgia bucomaxilofacial. 3. ed. São Paulo: Santos; 2006.

2. Sousa RR, Castro RD, Monteiro CH, Silva SC, Nunes AB. O paciente odontológico portador de diabete mellitus: Uma revisão de literatura. Pesq Bras Odontoped Clin Integr. 2003; 3(2):71-7.
3. Ettinger RL. Rational dental care: part 2. A case history. J Can Dent Assoc. 2006; 72(5):447-52.

4. Little JW, Rhodus NL. Dental management of the patient with cardiac arrhythmias: an update. Oral Surg Oral Med Oral Radiol Endod. 2003; 96(6):659-68.

5. Teixeira CS, Pasternak Júnior B, Silva-Sousa YTC, Perez DEC. Tratamento odontológico em pacientes com comprometimento cardiovascular. RSBO. 2008; 5(1):68-76.

6. Fiske J. Diabetes mellitus and oral care. Dent Update 2004; 31(4):190-6.

7. Carneiro Neto JN, Beltrame M, Souza IFA, Andrade JM, Silva JAL, Quintela KL. O paciente diabético e suas implicações para a conduta odontológica. Rev Dentística on-line. 2012; 11(23):11-18

8. Souza MOF, Perez ARHS, Souza TOF, Martins MAT, Bussadori SK, Fernandes KPS et al. Incidência de alterações sistêmicas e uso de medicamentos em pacientes atendidos em clínica odontológica Conscientiae Saúde 2007; 6(2):305-11.

9. Santos Melo ECS, Dantas CCBL, Barbosa AF. Manejo clínico de pacientes cardiopatas em periodontia revisão de literatura. R Periodontia. 2009; 19(4):30-6.

10. Gaetti-Jardim EC, Pereira FP, Fattah CMRS, Aranega AM. Prevalência e perfil epidemiológico das alterações sistêmicas em pacientes atendidos pelo serviço de cirurgia e traumatologia buco-maxilo-facial da Faculdade de Odontologia do Campos de AraçatubaUNESP. Rev Odontol UNESP. 2008; 37(2):191-6.

11. Carvalho PSP, Mosele OL. Ocorrência de enfermidades ou condições sistêmicas detectadas após avaliação préoperatória da saúde de 2.475 pacientes. Implant News. 2006; 3:346-52.

12. Queiroz TP, Marques DO, Santos PL, Saraiva HC, Esteves JC, Hochuli-Vieira E. Prevalência de alterações sistêmicas em pacientes atendidos na disciplina de cirurgia e traumatologia bucomaxilofacial do curso de odontologia da UNIARA. Rev Odontol. UNESP. 2012; 41(3):154-9.

13. Sonis ST, Fazio RC, Fang L. Princípios e prática de medicina oral. 2. ed., Rio de Janeiro: Guanabara Koogan; 1996.

14. Santos TS, Acevedo CR, Melo MCR, Dourado E. Abordagem atual sobre hipertensão arterial sistêmica no atendimento odontológico. Odontologia. Clín.-Científ Recife. 2008; 8(2):105-9.

15. Lima-Costa MF, Peixoto SV, Firmo JOA. Validade da hipertensão arterial auto-referida e seus determinantes (Projeto Bambuí). Rev Saúde Pública. 2004; 38(5):637-42.

16. Guevara HG, Mónaco GL, Rivero CS, Vasconcellos V, Souza DP, Raitz R. Manejo odontológico em pacientes com doença renal crônica. RBCS. 2014; 12(40):74-81.

17. Diretrizes brasileiras para o diagnóstico, tratamento e prevenção da febre reumática. Arq Bras Cardiol.2009;93(3 supl.4):1-18

18. Conrado VC, de Andrade J, de Angelis GA, de Andrade AC, Timerman L, Andrade MM et al. Cardiovascular Effects of Local Anesthesia with Vasoconstrictor during Dental Extraction in Coronary Patients. Arq.Bras.Cardiol. 2007; 88(5):446-52.

\section{CONFLITO DE INTERESSES}

Os autores declaram não haver conflitos de interesse. 


\section{AUTOR PARA CORRESPONDÊNCIA}

Rodrigo Capalbo da Silva

capalbo.rodrigo@gmail.com

Submetido em 30/05/2017

Aceito em 08/07/2017 